\title{
MVA-BN-Brachyury-TRICOM Vaccine
}

National Cancer Institute

\section{Source}

National Cancer Institute. MVA-BN-Brachyury-TRICOM Vaccine. NCI Thesaurus. Code C148400.

A cancer priming vaccine consisting of a proprietary version of the recombinant vaccinia viral vector, modified vaccinia Ankara-Bavarian Nordic (MVA-BN), encoding the human transcription factor and tumor-associated antigen (TAA) brachyury, and a triad of T-cell co-stimulatory molecules (TRICOM), which is comprised of the three human immuneenhancing co-stimulatory molecules B7-1, ICAM-1 and LFA-3, with potential immunostimulatory and antineoplastic activities. Upon subcutaneous administration of MVA-BN-brachyury vaccine, the vector expresses the brachyury protein. The expressed brachyury protein may induce a cytotoxic T-lymphocyte (CT L)-mediated immune response against tumor cells expressing brachyury. The MVA-BN-brachyury vaccine, which is a priming vaccine, is followed by multiple boosting doses of the fowlpox virus (FPV)-brachyury vaccine. The expression of brachyury, a member of the T-box family of transcription factors that is overexpressed in numerous cancer cell types, is correlated with increased epithelial-mesenchymal transition (EMT), cancer resistance, cancer progression and metastasis. TRICOM enhances antigen-specific T-cell activation. 